\title{
Age-related Changes in Triathlon Performances
}

Running head : Aging and triathlon performance

R. Lepers ${ }^{1}$, F. Sultana ${ }^{2}$, T. Bernard ${ }^{2}$, C. Hausswirth ${ }^{3}$, J. Brisswalter ${ }^{4}$

${ }^{1}$ University of Burgundy, Faculty of Sport Sciences, INSERM U887, BP 27877, 21078 Dijon Cedex France

${ }^{2}$ Department of Sport Ergonomic and Performance, UFR STAPS, University of Sud Toulon-Var, BP 132, 83957 La Garde Cedex, France

${ }^{3}$ Laboratory of Biomechanics and Physiology, French National Institute of Sport and Physical Education (INSEP), Avenue du Tremblay, 75012 Paris, France

${ }^{4}$ Laboratory of Human Motricity, Education and Health, University of Nice Sophia Antipolis, 261, Route de Grenoble, BP 3259, 06205 Nice, Cedex 03, France

Corresponding author :

\section{R. Lepers}

Université de Bourgogne

INSERM U 887

BP 27877

21078 Dijon cedex

France

Tel : +33.3.80.39.67.60

Fax : $+33.3+80.39 .67 .02$

Email : romuald.lepers@u-bourgogne.fr 


\title{
Age-related Changes in Triathlon Performances
}

\begin{abstract}
The aim of this study was twofold: i) to analyze age-related declines in swimming, cycling, and running performances for Olympic and Ironman triathlons, and ii) to compare age-related changes in these three disciplines between the Olympic and Ironman triathlons. Swimming, cycling, running and total time performances of the top 10 males between 20 and 70 years of age (in 5 years intervals) were analyzed for two consecutive world championships (2006 and 2007) for Olympic and Ironman distances. There was a lesser age-related decline in cycling performance $(\mathrm{P}<0.01)$ compared with running and swimming after 55 years of age for Olympic distance and after 50 years of age for Ironman distance. With advancing age, the performance decline was less pronounced $(\mathrm{P}<0.01)$ for Olympic than for Ironman triathlon in cycling (>55 years) and running ( $>50$ years), respectively. In contrast, an age-related decline in swimming performance seemed independent of triathlon distance. The age-related decline in triathlon performance is specific to the discipline, with cycling showing less declines in performance with age than swimming and running. The magnitude of the declines in cycling and running performance at Ironman distance is greater than at Olympic distance, suggesting that task duration exerts an important influence on the magnitude of the age-associated changes in triathlon performance.
\end{abstract}




\section{Introduction}

The age-related decline in endurance exercise performance is well documented. In general, peak endurance performance is maintained until $\sim 35$ years of age, followed by modest decreases until 50-60 years of age, and a progressively steeper decline thereafter [26]. However, most studies examining masters athletes have focused on running for different distances up to the marathon [28]. Fewer studies however, have examined the age-related decline in performance of other modes of locomotion such as swimming and cycling. Donato et al. [8] for example, suggested that the rates of decline in swimming performance with age were greater in a long-duration $(1500 \mathrm{~m})$ than in shortduration $(50 \mathrm{~m})$ events, because of a relatively smaller loss of anaerobic muscular power with age compared with cardiovascular endurance. Similarly, few studies have examined the age-related decline in cycling. Balmer et al. [1,2] for example, examined the age-related decline in cycling performance for an indoor 16-km time-trial. Cycling may show differences in the age-related decline than running because of the contraction types involved. Cycling is a non-weight bearing activity that employs predominantly concentric muscle activation compared with the stretch-shortening activity and eccentric activations during running. As older adults show different rates of decline during fatiguing contractions that involve eccentric as opposed to concentric activations, this may explain differences in the rate of decline in cycling versus running [3].

Triathlon, involving successive swimming, cycling and running sessions, represents an interesting model to examine the age-related changes in endurance performance in different locomotion modes. Official competition in triathlon varies from short distance named "Olympic triathlon" (1.5 km swim, $40 \mathrm{~km}$ cycling, and $10 \mathrm{~km}$ running) to long distance named also "Ironman triathlon” (3.8 km swim, $180 \mathrm{~km}$ cycling, and $42.2 \mathrm{~km}$ running) [4,14].

Bernard et al. [5] recently showed that for Olympic triathlon, performance times in the three disciplines declined with advancing age in a curvilinear manner although the pattern of decline was specific for each locomotion mode. Cycling for example, demonstrated the least age-associated decline compared to running and swimming. This observation suggests that physiological functional capacity and performance appears to be more resistant to biological aging in cycling than the two other 
disciplines. During a longer event, the age-related differences between cycling, running and swimming maybe even greater because the physiological and mechanical stresses that occur during Ironman triathlon are much greater compared to those encountered during the Olympic distance triathlon. For example, during an Ironman triathlon, energy expenditure ranges from 8500 to $11000 \mathrm{kcal}$ [13]. The number of impact forces during the Ironman marathon running are also much greater compared with a 10-km run. Because of the different physiological demands between Olympic and Ironman distance triathlons, the age-related declines in performance may differ between both distances. Therefore, the purpose of this study was: 1) to determine if there was a reduced rate of decline in cycling performance with advancing age compared to running and swimming for the Ironman triathlon, as has been previously shown for Olympic triathlon, and 2) to compare age-related changes in swimming, cycling, and running performances between the Olympic and Ironman distance triathlons.

\section{Materials \& Methods}

Swimming, cycling, running and total time performances of the top 10 non-elite males of 11 age groups were analyzed. The age groups distinguish the categories for each period of 5 years as follows with the corresponding associated code in brackets: 18-24 years (20), 25-29 years (25), 30-34 years (30), 35-39 years (35), 40-44 years (40), 45-49 years (45), 50-54 years (50), 55-59 years (55), 60-64 years (60), 65-69 years (65) and 70-74 years (70).

For the Olympic triathlon, the data set was obtained from the International Triathlon Union Web site: http.www.triathlon.org. Data were assessed for two consecutive world championships taking place in Lausanne (2006) and Hamburg (2007).

For the Ironman triathlon, the data set was obtained from World Triathlon Corporation Web site: http.www.ironmanlive.com. The world Ironman triathlon championship takes place every year in Kailua-Kona, Hawaii since 1981 [14]. Data were assessed for two consecutive Hawaii Ironman races in 2006 and 2007.

The mean time performance of the top 10 of the best performing age group for each individual discipline was very similar between 2006 and 2007 for both Olympic and Ironman triathlons (see 
results), so we pooled two years of data. For the Olympic and Ironman triathlons, the performances of 20 triathletes per age group (i.e. the top 10 of each age group in 2006 and in 2007) were considered. For each year and both distances, the swimming, cycling, running and overall time performances of each triathlete finishing in the top 10 of each age group was normalized to the mean time performance of the top 10 of the best performing age group. Thus, the age-related declines in performance were expressed using a ratio calculated between the individual and the mean time performances of the best performing age group for each mode of locomotion and total time.

\section{Statistical analysis}

Data are reported as means \pm SD with the text and displayed as means \pm SE in the figures. Two-way ANOVAs with repeated-measures on locomotion mode was used to compare performance ratio between modes of locomotion across ages for Olympic and Ironman triathlons. For each mode of locomotion and total time performance, two way ANOVAs (age x distance) was used to compare performance ratios between Olympic versus Ironman triathlon across age. Post hoc analysis (Tukey) was used to test for differences within the ANOVAs when appropriate. A significance level of $P<$ 0.05 was used to identify statistical significance.

\section{Results}

The mean time performances of the top 10 of the best performing age group for each discipline were very similar between 2006 and 2007 for both Olympic and Ironman triathlons. For Olympic triathlon, time performance (expressed as h:min:s) was : 0:20:42 \pm 0:01:24, 1:02:51 \pm 0:01:30, 0:35:55 \pm 0:01:19 in 2006 and 0:19:59 \pm 0:00:58, 1:00:21 \pm 0:00:34, 0:34:27 \pm 0:01:12 in 2007, for $1.5 \mathrm{~km}$ swimming, $40 \mathrm{~km}$ cycling and $10 \mathrm{~km}$ running, respectively. For Ironman triathlon, time performance (expressed as h:min:s) was: 1:00:58 \pm 0:04:44, 4:50:01 \pm 0:05:05, 3:04:54 \pm 0:05:50 in 2006 and 0:59:08 \pm 0:02:05, 4:56:47 \pm 0:07:52, 3:06:46 \pm 0:05:51 in 2007, for $3.8 \mathrm{~km}$ swimming, $180 \mathrm{~km}$ cycling and $42.2 \mathrm{~km}$ running, respectively. 
Swimming, cycling, running and total time performances for each age group for both Olympic and Ironman triathlons are presented in Table 1. The relative contribution of each part of the overall performance remains constant across the ages for both distances. For Olympic triathlon, the 1.5-km swimming, 40-km cycling, and 10-km running represent on average 17.2 \pm 0.6, $47.6 \pm 1.6,28.8 \pm 0.6$ $\%$ of the total time, respectively. For Ironman triathlon, the 3.8-km swimming, 180-km cycling, and 42-km running represent on average $11.3 \pm 0.4,52.3 \pm 1.3,35.1 \pm 1.0 \%$ of the total time, respectively.

\section{(Insert table 1)}

For both Olympic and Ironman distances, the performance ratios for each mode of locomotion decreased in a curvilinear manner with advancing age (Figures 1A and 1B). For both distances, there was an interaction of age and mode of locomotion $(\mathrm{P}<0.001)$. For Olympic distance, the performance ratio was significantly different $(\mathrm{P}<0.01)$ from the previous age group, from age group 40 for swimming, from age group 50 for running, and from age group 55 for cycling, respectively. Cycling performance ratio remained significantly $(\mathrm{P}<0.01)$ greater than swimming from age group 40 onwards, and from age group 45 cycling performance ratio remained significantly $(\mathrm{P}<0.01)$ greater than running. For Ironman distance, the performance ratio was significantly different $(\mathrm{P}<0.01)$ from the previous age group, from age group 45 for swimming and running, and from age group 50 for cycling. Cycling performance ratio remained significantly $(\mathrm{P}<0.01)$ greater than swimming from age group 45 onwards (except for age group 70), and from age group 50 cycling performance ratio remained significantly $(\mathrm{P}<0.01)$ greater than running.

(Insert Figures 1A \& 1B)

Comparisons of age related declines in swimming, cycling, running and total performance for Olympic and Ironman triathlons are presented in Figure 2. There was an interaction $(\mathrm{P}<0.001)$ of age and distance for cycling, running, and total event times, but not for swimming times. Age-related declines in performance were less $(\mathrm{P}<0.01)$ pronounced for Olympic compared with the Ironman 
distance for cycling, running and total time from the following age groups and above: 55, 50, and 55, respectively.

\section{(Insert Figure 2)}

\section{Discussion}

The main findings of the present study were i) a reduced age-related decline in cycling performance compared with running and swimming performances for both Olympic and Ironman distance triathlons, and ii) a smaller effect of age on cycling and running performances for Olympic than for Ironman distance triathlon, but a similar age-related decline in swimming performance for Olympic and Ironman distance triathlon.

\section{Methodological considerations}

With endurance events, the environment (temperature, humidity, precipitation) affects the outcome of the results. For Olympic triathlons taking place in Lausanne (2006) and Hambourg (2007), the temperature was around $20^{\circ} \mathrm{C}$ and $15^{\circ} \mathrm{C}$ respectively, whereas during the Hawaii Ironman in 2006 and 2007, the temperature was around $30^{\circ} \mathrm{C}$. Thus, the hotter conditions for the Ironman triathlon may have more greatly affected performance than for the Olympic triathlon. However, by normalizing the individual time performance by the mean time performance of the top 10 of the best performing age groups for both distances, the influence of environment was minimized when comparing Olympic and Ironman triathlon performances. Moreover, the results showed that the mean time performances of the best performing age groups for each individual discipline were very similar between 2006 and 2007 for both Olympic and Ironman triathlons.

The present paper focuses on the performances of male triathletes only. Potential differences in age-related decline in endurance performance between male and female may also occur, so it could be interesting to verify if similar results are obtained for female triathletes. However, for Ironman triathlon, data for female triathletes older than 65 years of age are limited. Moreover, unpublished data from our laboratory suggest that similar findings are observed for female triathletes i.e. a reduced age- 
related declines in cycling performance compared with running and swimming performances and a greater magnitude of age-related decline in cycling and running performance for the Ironman triathlon than for the Olympic triathlon.

The present data do not include performance of elite/professional triathletes. At the world Olympic triathlon championships, elite triathletes raced a different course with different rules (drafting allowed for the elite athletes and forbidden for the age groupers). At the world Ironman triathlon championships, elite triathletes raced the same course but started 15 minutes before the age groups (drafting forbidden for both the elite and age groups).

\section{Age related differences in locomotion mode}

For both Olympic and Ironman triathlons, there was a lesser age-related decline in cycling performance compared with running and swimming performances. Bernard et al. [5] had previously reported a less pronounced age-related decline in cycling performance than the other locomotion modes but for Olympic triathlon only. Thus, the question of whether there was a better maintenance of cycling performance or a greater decline in running and swimming performances with advancing age can be raised. One explanation for the different age-related declines in cycling and running may involves the mechanical power required by these disciplines. Mechanical power output in running depends on the velocity $(\mathrm{P}=\mathrm{k} . \mathrm{V})$ while it depends on third power of velocity in cycling $\left(\mathrm{P}=\mathrm{k} . \mathrm{V}^{3}\right)$. If we assume that the changes in aerobic capacity (e.g. $\mathrm{VO}_{2} \max$ ) with age are directly related to the decline in mechanical power, a similar reduction in power output for running and cycling with advancing age would induce a lower reduction in cycling velocity than running velocity. This may explain, in part, why the magnitude of the decrease in cycling performance with age was less than that for running.

Running also involves stretch-shortening cycles (SSC) with eccentric muscle actions, while cycling is a non weight-bearing activity with dominant concentric muscle actions. Fast twitch fibres however, atrophy more than slow twitch fibres with age [10]. Because, fast twitch fibres seem more susceptible to damage than slow twitch fibres during SSC, the greater reduction in running 
performance compared with cycling could therefore be related to muscle typology changes with age [30]. However the changes in muscle fibre type distribution i.e. the percentage of type I muscle fibres with advancing age seem less pronounced in well-trained masters athletes compared to untrained older adults [29]. Moreover, in terms of muscle damage, although some animal studies have shown that recovery from eccentric exercise-induced muscle damage is lessened in older subjects compared with the young, the results from humans studies are not as clear [7]. Results might differ among muscle age groups but there is no evidence that the muscles of older triathletes would be more susceptible to muscle damage than muscles of young triathletes.

Findings for competitive long-distance runners indicate that the decline in running times parallel the age-related reductions in maximal oxygen uptake and in lactate threshold $[11,20]$. The contributions of these respective physiological factors to age-related declines in cycling performance however, are unknown at present. Indeed, very few studies are available on the effect of age on cycling performance. Balmer et al. [1,2] recently characterized the decline in maximal oxygen consumption and maximal aerobic power with age in cycling. However, the relative intensity expressed as a percentage of maximal heart rate or maximal oxygen uptake as well as economy was not affected by age during a $\sim 20$ min time trial. Thus, it is tempting to speculate that the lesser decline in cycling performance with advancing age could be due to a lesser reduction in lactate threshold and/or economy compared with running.

An alternative explanation for the smaller age-related decline performance in cycling compared to running is the maintenance of a relative greater exercise training "stimulus" in cycling. It has been shown that an overall reduction in the exercise training "stimulus" occurs with advancing age $[21,26]$. In running, the decline in exercise performance with age has been partly attributed to an increased incidence of orthopedic injuries, which would limit running training volume of many older athletes [20,12]. It is possible that cycling performance was influenced to a much lesser extent by this factor. Moreover, it has been shown that protein synthesis rate is decreased in older subjects compared with young subjects that could limit the running training volume in older triathletes where muscle damage occurs [27]. With advancing age triathletes would tend to spend more training time on cycling than running, as a consequence of changes in physical factors (e.g. increased prevalence of injuries). 
Unfortunately, change in training volume in the three disciplines with age was unknown in the present study. Further prospective studies would be necessary to quantify the changes in training volume in triathletes with age because training history is an important aspect of performance status at an older age.

For both Olympic and Ironman triathlons, swimming and running performances decline in a similar manner until 65-70 years. This result differs from those of Tanaka and Seals [25] who found a smaller reduction in swimming than in running performances with advancing age. These authors suggested that the two hypotheses to explain this task-specific difference were a greater dependence on biomechanical techniques for swimming than for running, and a greater prevalence of orthopaedic injuries during running with advancing age, which could limit the training volume compared with swimming [9]. However, Tanaka and Seals [25] analysed the changes in swimming performance with advancing age for distance $\leq 1500 \mathrm{~m}$ in swimming pool conditions that differ greatly from open-water conditions in triathlon. Open-water conditions might include currents and waves especially in the ocean (3.8 km swim at Hawaii Ironman); moreover in triathlon, the swimming start is a mass start, a stressful situation in which triathletes may become disturbed, especially when they are not very good swimmers. In addition, triathletes may not swim at the maximal speeds because they have to cycle and run following the swim. All these factors might explain a greater reduction in swimming performances with advancing age in triathlon compared to conventional swimming in the pool and therefore the absence of differences between changes in running and swimming performance with advancing age in triathlon.

\section{Task duration and age-related changes in performance}

Age-related changes in swimming performance were not influenced by task duration; the magnitudes of decreases in swimming performance were similar for Olympic versus Ironman triathlon. Swimming is the first discipline during a triathlon, so triathletes perform it without suffering from accumulated fatigue in contrast to the cycling and running disciplines. On average, swimming performance declines by $\sim 15 \%$ at 50 years old, and reaches $\sim 38 \%$ by 70 years of age. Previous studies showed no effect of task duration for shorter swimming distance events. For example, Tanaka \& Seals 
[24] found no significant differences in the magnitude of performance decline with age ( $\sim 32 \%$ at 70 yrs age) among distance events ranging from 100 to $1500 \mathrm{~m}$ in pool. The lower performance decline observed by these authors for the same age (70 yrs old) compared with triathlon data might be due the differences between the water conditions: calm in swimming pool versus stochastic in open water for triathlon.

In contrast to swimming, the magnitude of the declines in cycling and running performances with advancing age at Olympic triathlon was less pronounced than at Ironman triathlon. It is not clear why task duration in triathlon exerts an influence on age-related declines in cycling and running performance. For the 70-74 yrs age group, the finish time was $~ 3$-h for the Olympic triathlon versus 15-h for Ironman triathlon. Certainly the Ironman triathlon induces greater neuromuscular fatigue in cycling and running compared with Olympic triathlon [15,18]. Furthermore, muscle damage during 10-k run of Olympic distance is limited compared to that occurring during the Ironman marathon $[17,23]$. Endurance exercise increases the use of endogenous fuels to provide energy for working muscles. However, resting muscle glycogen concentration is diminished in older humans [6\}], and hepatic gluconeogenic and glyconeogenic capabilities have been found to decline with age [19]. Older subjects oxidize more glucose and less fat during moderate intensity exercise [16]. This shift in substrate use is presumably caused by age-related changes in skeletal muscle, including decreased skeletal muscle respiratory capacity. Similarly, Sial et al. [22] found that compared with young adults, fat oxidation was lower in older adults during endurance exercise performed at either the same absolute or relative intensity. Changes in substrates availability and utilisation with advancing age could explain in part the task duration effect on triathlon performances at least in cycling and running.

\section{Conclusion}

This cross sectional study found that the age related declines in cycling performance are lesser than in swimming and running at both Olympic and Ironman triathlons. These findings indicate that age related declines in endurance performance are specific to the locomotor task although the cause for the task-specificity is not clear. In contrast to swimming, the magnitudes of age-related declines in cycling and running performance for the Ironman triathlon are greater than for the Olympic triathlon, 
suggesting that task duration exerts an important influence on the age-associated changes in triathlon performance. Further studies examining the changes in training volume and physiological characteristics of older triathletes are required to better understand the age-associated changes in triathlon performance and will provide valuable information for understanding the way to maintain physical capacity and performance with advanced age.

\section{References}

1. Balmer J, Bird S, Davison R. Indoor 16.1-km time-trial performance in cyclists aged 25- 63 years. J Sports Sci 2008; 26: 57-62

2. Balmer J, Bird S, Davison R, Lucia A. Effect of age on 16.1-km time-trial performance. J Sports Sci 2008; 26: 197-206

3. Baudry S, Klass M, Pasquet B, Duchateau J. Age-related fatigability of the ankle dorsiflexor muscles during concentric and eccentric contractions. Eur J Appl Physiol 2007; 100: 515-525

4. Bentley DJ, Millet GP, Vleck VE, McNaughton LR. Specific aspects of contemporary triathlon: implications for physiological analysis and performance. Sports Med 2002; 32: 345-359

5. Bernard T, Sultana F, Lepers R, Hausswirth C, Brisswalter J Age related decline in Olympic triathlon performance: effect of locomotion mode. Exp Aging Res 2010; 36 :1-15

6. Cartee GDAging skeletal muscle: response to exercise. Exerc Sport Sci Rev 1994; 22: 91-120

7. Chapman DW, Newton M, McGuigan MR, Nosaka K. Comparison between old and young men for responses to fast velocity maximal lengthening contractions of the elbow flexors. Eur J Appl Physiol 2008; 104: 531-539

8. Donato AJ, Tench K, Glueck DH, Seals DR, Eskurza I, Tanaka H. Declines in physiological functional capacity with age: a longitudinal study in peak swimming performance. J Appl Physiol 2003; 94: 764-769

9. Egermann M, Brocai D, Lill C A, Schmitt H. Analysis of injuries in long-distance triathletes. Int J Sports Med 2003; 24: 271-276 
10. Faulkner JA, Davis CS, Mendias CL, Brooks SV. The aging of elite male athletes: age-related changes in performance and skeletal muscle structure and function. Clin J Sport Med 2008; 18: $501-507$

11. Fuchi T, Iwaoka K, Higuchi M, Kobayashi S. Cardiovascular changes associated with decreased aerobic capacity and aging in long-distance runners. Eur J Appl Physiol 1989; 58: 884-889

12. Kallinen M, Markku A. Aging, physical activity and sports injuries. An overview of common sports injuries in the elderly. Sports Med 1995; 20: 41-52

13. Kreider RB. Physiological considerations of ultraendurance performance. Int J Sport Nutr 1991; $1: 3-27$

14. Lepers R. Analysis of Hawaii ironman performances in elite triathletes from 1981 to 2007. Med Sci Sports Exerc 2008; 40: 1828-1834

15. Lepers R, Maffiuletti NA, Rochette L, Brugniaux J, Millet GY. Neuromuscular fatigue during a long-duration cycling exercise. J Appl Physiol 2002; 92: 1487-1493

16. Mittendorfer B, Klein S. Effect of aging on glucose and lipid metabolism during endurance exercise. Int J Sport Nutr Exerc Metab 2001; 11: S86-91

17. Neubauer O, König D, Wagner KH. Recovery after an Ironman triathlon: sustained inflammatory responses and muscular stress. Eur J Appl Physiol 2008; 104: 417-426

18. Place N, Lepers R, Deley G, Millet GY. Time course of neuromuscular alterations during a prolonged running exercise. Med Sci Sports Exerc 2004; 36: 1347-1356

19. Podolin DA, Pagliassotti MJ, Gleeson TT, Mazzeo RS. Influence of endurance training on the agerelated decline in hepatic glyconeogenesis. Mech Ageing Dev 1994; 75: 81-93

20. Pollock ML, Foster C, Knapp D, Rod JL, Schmidt DH. Effect of age and training on aerobic capacity and body composition of master athletes. J Appl Physiol 1987; 62: 725-731

21. Pollock ML, Mengelkoch LJ, Graves JE, Lowenthal DT, Limacher MC, Foster C, Wilmore JH. Twenty-year follow-up of aerobic power and body composition of older track athletes. J Appl Physiol 1997; 82: 1508-1516

22. Sial S, Coggan AR, Hickner RC, Klein S. Training-induced alterations in fat and carbohydrate metabolism during exercise in elderly subjects. Am J Physiol 1998; 274: E785-790 
23. Suzuki K, Peake J, Nosaka K, Okutsu M, Abbiss CR, Surriano R, Bishop D, Quod MJ, Lee H, Martin DT, Laursen PB. Changes in markers of muscle damage, inflammation and HSP70 after an Ironman Triathlon race. Eur J Appl Physiol 2006; 98: 525-534

24. Tanaka H, Seals DR. Age and gender interactions in physiological functional capacity: insight from swimming performance. J Appl Physiol 1997; 82: 846-85

25. Tanaka H, Seals DR. Invited Review: Dynamic exercise performance in masters athletes: insight into effects of primary human aging on physiological functional capacity. J Appl Physiol 2003; 95: 2152-2162

26. Tanaka H, Seals DR. Endurance exercise performance in Masters athletes: age-associated changes and underlying physiological mechanisms. J Physiol 2008; 586: 55-63

27. Toth MJ, Matthews DE, Tracy RP, Previs MJ. Age-related differences in skeletal muscle protein synthesis: relation to markers of immune activation. Am J Physiol 2005;288:E883-891

28. Trappe S. Marathon runners: how do they age? Sports Med 2007; 37: 302-305

29. Trappe SW, Costill DL, Fink WJ, Pearson DR. Skeletal muscle characteristics among distance runners: a 20-yr follow-up study. J Appl Physiol 1995; 78 :823-829

30. Vijayan K, Thompson JL, Norenberg KM, Fitts RH, Riley DA. Fiber-type susceptibility to eccentric contraction-induced damage of hindlimb-unloaded rat AL muscles. J Appl Physiol 2001; 90: 770-776 


\section{Legends}

\section{Figure 1}

Age-related declines in swimming, cycling and running performances for the Olympic (Panel A) and Ironman triathlons (Panel B), (mean \pm SE). For Olympic triathlon, the performance ratio was significantly different $(\mathrm{P}<0.01)$ from the previous age group, from age group 40 for swimming, from age group 50 for running, and from age group 55 for cycling, respectively. Cycling performance ratio remained significantly $(\mathrm{P}<0.01)$ greater than swimming from age group 40 onwards, and from age group 45 cycling performance ratio remained significantly $(\mathrm{P}<0.01)$ greater than running. For Ironman triathlon, the performance ratio was significantly different $(\mathrm{P}<0.01)$ from the previous age group, from age group 45 for swimming and running, and from age group 50 for cycling. Cycling performance ratio remained significantly $(\mathrm{P}<0.01)$ greater than swimming from age group 45 onwards (except for age group 70), and from age group 50 cycling performance ratio remained significantly $(\mathrm{P}<0.01)$ greater than running.

\section{Figure 2}

Age-related declines in swimming, cycling, running and total performances for Olympic and Ironman triathlons (mean $\pm \mathrm{SE}$ ). There was no distance effect for swimming performance. However, agerelated decline in cycling, running, and total performance was less pronounced for Olympic triathlon. *: $P<0.01$ : Significantly different from Ironman triathlon for the same age group. 

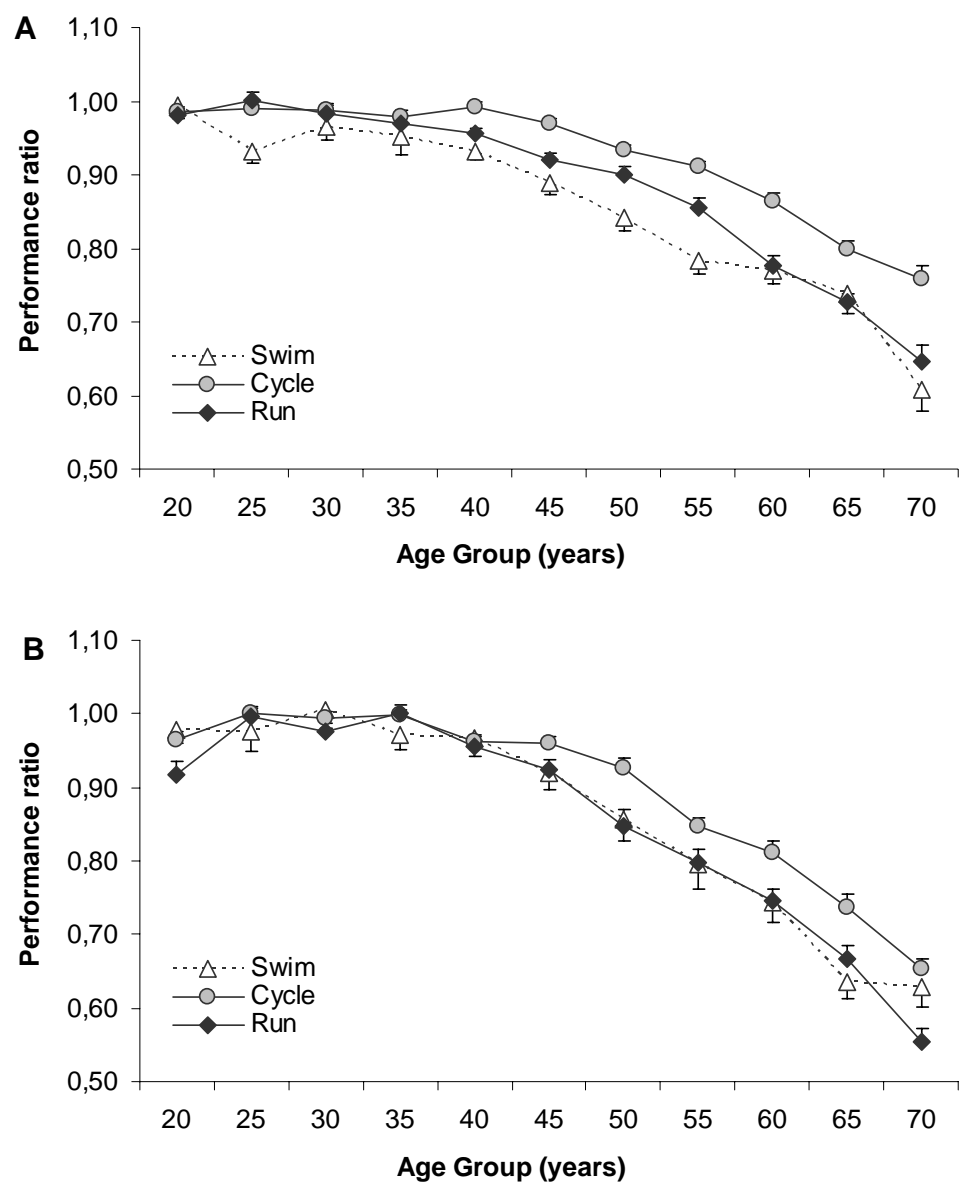

Figure 1 

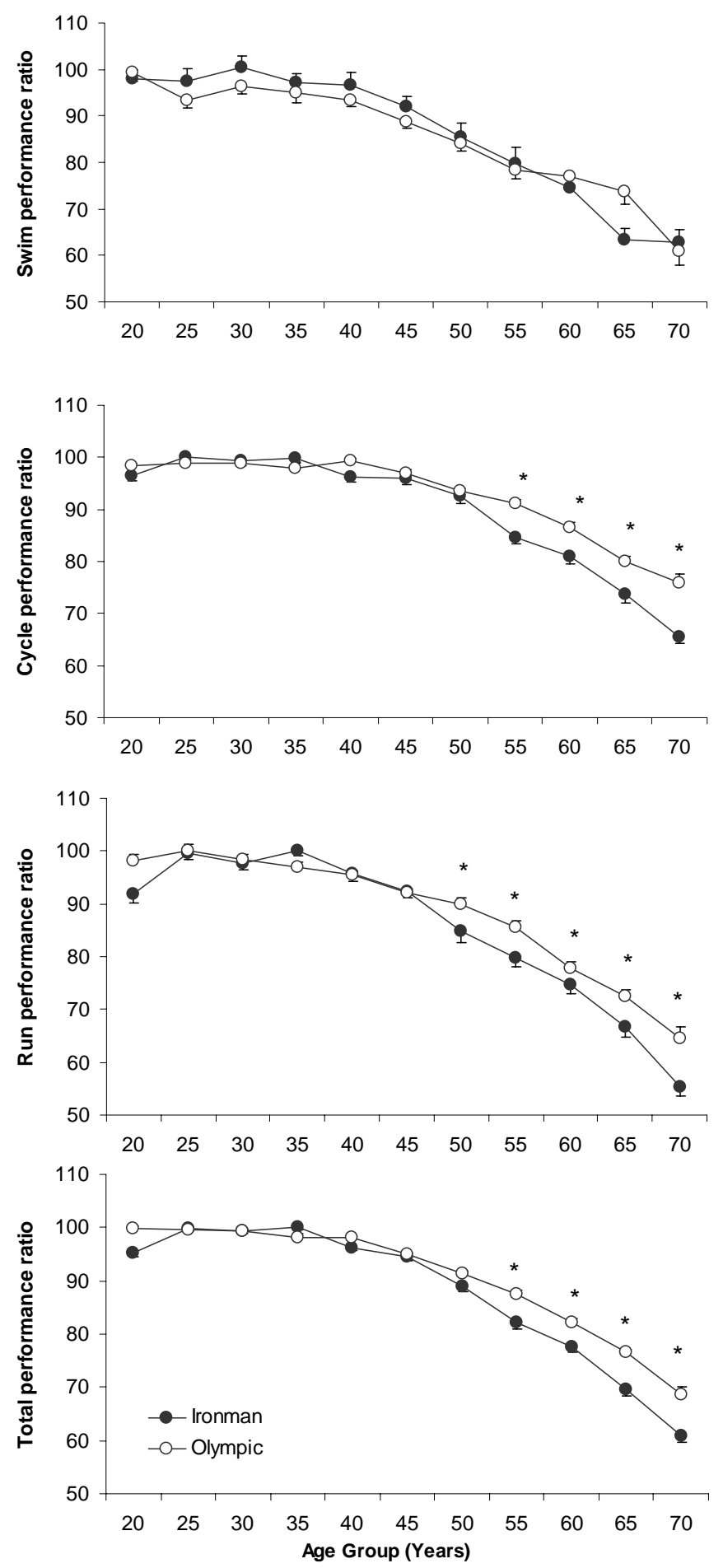

Figure 2 
Age groups (years)

\begin{tabular}{|c|c|c|c|c|c|c|c|c|c|c|c|}
\hline & \multicolumn{11}{|c|}{ Age groups (years) } \\
\hline & $18-24$ & $25-29$ & $30-34$ & 35-39 & $40-44$ & $45-49$ & $50-54$ & 55-59 & $60-64$ & 65-69 & $70-74$ \\
\hline \multicolumn{12}{|l|}{ Olympic Triathlon } \\
\hline \multirow[t]{2}{*}{$1.5 \mathrm{~km}$ Suim (min:s) } & $20: 20$ & $21: 45$ & 21:02 & $21: 24$ & $21: 44$ & $22: 50$ & 24:07 & $25: 58$ & $26: 25$ & $27: 44$ & $34: 00$ \\
\hline & \pm 1:19 & $\pm 1: 15$ & $\pm 1: 11$ & $\pm 1: 39$ & $\pm 0: 51$ & $\pm 1: 12$ & $\pm 1: 29$ & $\pm 1: 54$ & $\pm 2: 09$ & $\pm 3: 03$ & $\pm 5: 14$ \\
\hline 40 km Cycle (h:min:s) & $\pm 2: 03$ & $\pm 1: 59$ & $\pm 1: 33$ & $\pm 1: 50$ & $\pm 2: 24$ & $\pm 2: 21$ & $\pm 2: 34$ & $\pm 2: 36$ & $\pm 3: 46$ & $\pm 3: 30$ & $\pm 7: 11$ \\
\hline \multirow[t]{2}{*}{10 km Run (min:s) } & $35: 56$ & $35: 11$ & $35: 48$ & $36: 20$ & $36: 50$ & $38: 16$ & 39:08 & $41: 13$ & $45: 24$ & 48:34 & $55: 13$ \\
\hline & $\pm 1: 44$ & $\pm 1: 26$ & $\pm 1: 29$ & $\pm 1: 37$ & $\pm 1: 02$ & $\pm 1: 31$ & $\pm 1: 50$ & $\pm 2: 00$ & $\pm 2: 42$ & $\pm 2: 47$ & $\pm 7: 29$ \\
\hline Total (h:min:s) & 2:05:56 & 2:06:15 & 2:06:22 & $2: 07: 53$ & 2:08:10 & $2: 12: 24$ & $2: 17: 30$ & 2:23:37 & $2: 32: 57$ & $2: 44: 10$ & 3:04:15 \\
\hline \multicolumn{12}{|l|}{ Ironman Triathlon } \\
\hline \multirow[t]{2}{*}{3.8 km Suim (h:min:s) } & 1:01:38 & 1:02:04 & 1:00:07 & 1:02:09 & 1:02:35 & 1:05:44 & 1:11:04 & 1:16:51 & 1:21:49 & 1:35:51 & 1:37:26 \\
\hline & $\pm 4: 01$ & $\pm 5: 22$ & $\pm 4: 30$ & \pm 4:05 & $\pm 5: 23$ & \pm 5:00 & $\pm 8: 05$ & $\pm 10: 35$ & $\pm 9: 36$ & $\pm 10: 46$ & $\pm 13: 34$ \\
\hline \multirow[t]{2}{*}{180 km Cycle (h:min:s) } & 5:04:17 & 4:53:24 & $4: 55: 26$ & 4:54:01 & 5:05:25 & 5:06:15 & $5: 17: 44$ & $5: 47: 18$ & 6:03:24 & $6: 40: 18$ & 7:30:09 \\
\hline & $\pm 10: 08$ & $\pm 7: 32$ & $\pm 8: 46$ & $\pm 6: 04$ & $\pm 8: 15$ & $\pm 11: 11$ & $\pm 15: 10$ & $\pm 16: 37$ & $\pm 22: 58$ & $\pm 32: 02$ & $\pm 25: 54$ \\
\hline \multirow[t]{2}{*}{42 km Run (h:min:s) } & 3:23:08 & 3:07:03 & 3:10:37 & 3:05:58 & 3:14:52 & $3: 21: 41$ & 3:40:31 & 3:53:51 & 4:10:26 & 4:40:56 & 5:38:55 \\
\hline & $\pm 11: 12$ & $\pm 6: 43$ & $\pm 7: 04$ & $\pm 6: 06$ & $\pm 9: 11$ & $\pm 9: 13$ & $\pm 17: 50$ & $\pm 15: 38$ & $\pm 16: 42$ & $\pm 23: 51$ & $\pm 33: 27$ \\
\hline Total (h:min:s) & 9:35:26 & 9:08:22 & 9:11:57 & 9:07:53 & 9:29:23 & 9:40:27 & 10:16:40 & 11:07:21 & 11:46:38 & 13:09:27 & 15:02:17 \\
\hline
\end{tabular}

Table 1

Shimming, cycling, running, and total performance times for the top 10 males in each age group at the world triathlon championships. For Olympic triathlon, data from Lausanne (2006) and

Hamburg (2007) were averaged. For Ironman triathlon, data from Hawaii Ironman in 2006 and 2007 were averaged. Values are mean \pm SD 